\title{
Proactive Consultation by a Clinical Pathologist Prevents Diagnostic Delay in Hematological Malignancies
}

\author{
Chih-Chun Chang ${ }^{1 \&}$, Ming-Jang Su${ }^{1 \&}$, Jung-Li Ho ${ }^{1 \&}$, Jen-Tang Sun ${ }^{2}$,Huang-Wen \\ Tsai $^{3}$, Hui-Fei Tang ${ }^{1}$, Fang-Yeh Chu ${ }^{1,4,5 *}$
}

\begin{abstract}
Background: Diagnostic difficulties in hematological malignancies may lead to unacceptably prolonged help-seeking to diagnostic interval as well as increased complications and poor outcomes. Proactive consultation by a clinical pathologist (PCCP) may help clinical diagnosis and therapeutic strategy. Hence, the aim of this investigation was to evaluate the effect of PCCP on the help-seeking to diagnostic interval in hematological cancer cases. Materials and Methods: From January to November, 2015, abnormal results of hematological laboratory testing with added laboratory comment were selectively screened out, and patients with such abnormalities in hematological laboratory testing and accompanied laboratory comment with PCCP were enrolled. Results: A total of 125 aberrant results of hematological laboratory testing were given with accompanied laboratory comments with PCCP and 40.8\% ( $n=51)$ of these patient-oriented comments had an effect on clinical diagnosis and therapeutic strategy. Twelve of the subjects belonged to newly diagnosed hematological malignancies with the assistance of PCCP, and the help-seeking to diagnostic interval was also shortened from 42 days to 26 days in chronic lymphoid leukemia (CLL), from 83 days to 11 days in multiple myeloma (MM), and from 128 days to 15 days in myelodysplastic syndrome/myeloproliferative neoplasm (MDS/MPN). During the monitoring interval, neither complication events nor deaths were reported in the study group. Conclusions: It was seemingly that PCCP prevented diagnostic delay in hematological malignancies via shortening the help-seeking to diagnostic interval, particularly in CLL, MM and MDS/MPN cases. PCCP can be considered to play an essential role in prompt establishment of diagnosis in hematological malignancies for those who newly present.
\end{abstract}

Keywords: Active laboratory consultation - proactive consultation by clinical pathologist - hematological malignancies

Asian Pac J Cancer Prev, 17 (3), 1019-1022

\section{Introduction}

It was not easy to establish an early diagnosis for hematological cancers such as leukemia, lymphoma and myeloma, because of the ill-defined symptoms at the initial phase. Hence, there might be multiple primary healthcare consultations before referral to the specialties, leading to excessive intervals between symptom onset, help-seeking and diagnosis (Ong et al., 1995; Kariyawasan et al., 2007; Friese et al., 2009; Irfan et al., 2009; Summerfield et al., 2000; Elliss-Brookes et al., 2012; Lyratzopoulos et al., 2013). It was also reported that delayed diagnosis was associated with increased complication events in patients with hematological cancers (Summerfield et al., 2000; Kariyawasan et al., 2007). Besides, more than 60 subtypes of hematological cancers had been identified over these decades and many of them were characterized with unique clinical presentations and prognosis, making it difficult for further differential diagnosis of hematological malignancies from benign disorders (Howell et al.,
2013). As a consequence, it had been great challenges for clinicians to diagnose hematological cancers promptly.

Active laboratory consultation, first described and clinically practiced by clinical pathologists in Japan, was mainly composed of laboratory comments and liaison service for selectively abnormal results of laboratory testing (Kuwajima et al., 1997). It was reported that such proactive consultation by clinical pathologist (PCCP) could help clinical diagnosis and therapeutic strategy (Kuwajima et al., 1997; Kuwajima et al., 1998). Therefore, we conducted this study to evaluate the effect of PCCP on the help-seeking to diagnostic interval in hematological cancers.

\section{Materials and Methods}

From January to November, 2015, selectively abnormal results of hematological laboratory testing with added laboratory comment were screened out, and patients with such abnormalities in hematological 
laboratory testing and accompanied laboratory comment with PCCP were enrolled. The clinical condition was re-confirmed via the medical chart review or history taking and physical examination from the patient, and abnormal results of hematological laboratory testing were interpreted with patient-oriented laboratory comment written and validated by the clinical pathologist. After the validation of laboratory comment, the patient would be followed up for 2 months to estimate if there was an effect on clinical diagnosis and therapeutic strategy. In these laboratory data with PCCP, patients being suspected to suffer from hematological malignancies at the first time were selected out for further analysis. The hematological malignancies were mainly categorized into acute myeloid leukemia (AML), acute lymphoid leukemia (ALL), chronic myeloid leukemia (CML), chronic lymphoid leukemia (CLL), multiple myeloma (MM), and myelodysplastic syndrome/myeloproliferative neoplasm (MDS/MPN). The interval from validation for the abnormal results of hematological laboratory testing to the diagnostic verification by evidence of anatomical or molecular pathology and initiation of cancer-specific therapy was recorded as the help-seeking to diagnostic interval. The results were analyzed in comparison with the data adapted from previous studies (Kuwajima et al., 1998; Howell et al., 2013).

For statistical analysis, data were expressed as the median with interquartile range (IQR) or number with percentage. The statistical software, SPSS, was used for analysis in the study (version 15.0; SPSS Inc., Chicago, USA).

\section{Results}

During the study interval, a total of 125 aberrant results of hematological laboratory testing were given with accompanied laboratory comments with PCCP according to patient orientation. Of these, $40.8 \%(n=51)$ of patient-oriented comments had an effect on clinical diagnosis and therapeutic strategy. There were 12 subjects who belonged to the newly diagnosed hematological malignancies, including 3 cases of AML, 1 cases of ALL, 1 case of concurrent AML and ALL, 2 cases of CML, 1 case of CLL, 1 case of MM and 3 cases of MDS/MPN, and were selected out for further evaluation. The baseline characteristics of these subjects, including patient age, gender, diagnosis, the help-seeking to diagnostic interval and outcome were listed in Table 1. After a 2-month follow-up, all patients received optimal treatment according to respective diagnosis and were alive during the monitoring interval.

Table 2 showed the comparison of the median help-seeking to diagnostic interval in hematological malignancies from the perspective of patients in our results related to PCCP with the data adapted from the previous study, which was currently known as the most comprehensive investigation asking subjects across all subtypes of hematological cancers with the largest scale (Howell et al., 2013). It was seemingly that the helpseeking to diagnostic interval was apparently shortened

Table 2. Comparison of the Median Help-seeking to Diagnostic Interval in Hematological Malignancies from the Perspective of Patients between our Experience of PCCP and the Previous Study (Howell et al., 2013)

\begin{tabular}{lcc}
\hline \multicolumn{1}{c}{ Subtypes } & $\begin{array}{c}\text { FEMH experience } \\
\text { (days) }\end{array}$ & $\begin{array}{c}\text { Howell et al., } \\
2013 \text { (days) }\end{array}$ \\
\hline AML & $8(\mathrm{n}=4)$ & $10(5-32)$ \\
CML & $11(\mathrm{n}=2)$ & $9(5-32)$ \\
ALL & $10(\mathrm{n}=2)$ & $12.5(3-32)$ \\
CLL & $26(\mathrm{n}=1)$ & $42(12-88)$ \\
MM & $11(\mathrm{n}=1)$ & $83(34-167)$ \\
MDS/MPN & $15(\mathrm{n}=3)$ & $128(27-840)$ \\
\hline
\end{tabular}

The data were expressed as the median (case number) or (interquartile range). AML, acute myeloid leukemia; ALL, acute lymphoid leukemia; CML, chronic myeloid leukemia; CLL, chronic lymphoid leukemia; MM, multiple myeloma; MDS/MPN, myelodysplastic syndrome/ myeloproliferative neoplasm

Table 1. The Baseline Characteristics of patients who had Newly Diagnosed Hematological Malignancies with the Assistance of PCCP

\begin{tabular}{|c|c|c|c|c|}
\hline Patient no. & Age (years)/Gender (M/F) & Diagnosis & $\begin{array}{c}\text { The help-seeking to diagnostic } \\
\text { interval (days) }\end{array}$ & Outcome \\
\hline 1 & $71 / \mathrm{M}$ & AML & 4 & Alive \\
\hline 2 & $51 / \mathrm{F}$ & AML & 20 & Alive \\
\hline 3 & $67 / \mathrm{F}$ & AML & 3 & Alive \\
\hline 4 & $27 / \mathrm{M}$ & concurrent AML and ALL & 12 & Alive \\
\hline 5 & $48 / \mathrm{M}$ & ALL & 8 & Alive \\
\hline 6 & 26/M & CML & 5 & Alive \\
\hline 7 & $62 / \mathrm{M}$ & CML & 17 & Alive \\
\hline 8 & $64 / \mathrm{M}$ & CLL & 26 & Alive \\
\hline 9 & $75 / \mathrm{M}$ & $\mathrm{MM}$ & 11 & Alive \\
\hline 10 & $91 / \mathrm{F}$ & MDS/MPN & 12 & Alive \\
\hline 11 & 64/M & MDS/MPN & 20 & Alive \\
\hline 12 & 65/M & MDS/MPN & 15 & Alive \\
\hline
\end{tabular}

AML, acute myeloid leukemia; ALL, acute lymphoid leukemia; CML, chronic myeloid leukemia; CLL, chronic lymphoid leukemia; MM, multiple myeloma; MDS/MPN, myelodysplastic syndrome/myeloproliferative neoplasm 
from 42 days to 26 days in CLL, from 83 days to 11 days in MM and from 128 days to 15 days in MDS/MPN, with the assistance of PCCP. Besides, it seemed that there was no obvious difference of the help-seeking to diagnostic interval in AML (from 10 days to 8 days), ALL (from 12.5 days to 10 days) and CML (from 9 days to 11 days), although the help-seeking to diagnostic interval in some of the hematological malignancies was slightly shortened with the presence of PCCP.

\section{Discussion}

Our main finding suggested that, with the assistance of PCCP, the median help-seeking to diagnostic interval was shortened in hematological malignancies, particularly in CLL, MM and MDS/MPN. PCCP was considered to play an essential role in the promptly diagnostic establishment in hematological malignancies for those who were newly diagnosed.

The delayed establishment of diagnosis for hematological malignancies could be associated with increased complications in such populations, particularly in MM (Friese et al., 2009). And in Great Britain, it was estimated that the excess of annual deaths due to hematological malignancies could be up to hundreds of subjects, which was considered to be potentially avoidable (Abdel-Rahman et al., 2009). Recently, it was suggested that timely diagnosis might improve the prognosis of such populations (Thomson et al., 2009). However, diagnostic difficulties were often encountered in clinical, as the prodromata of hematological cancers in freshly diagnosed patients could be of broad spectrum and non-specific (Howell et al., 2008). Therefore, it was not easy for clinicians to differentiate benign disorders and self-limiting situations as well as identify the potential malignancies, depending on the clinical presentations. Timely diagnosis in hematological malignancies still posed great challenges for clinicians.

It was believed that the clinical parameters, including the examination of complete blood count, white cell differential count, and other diagnostic testing results, could be attributed to rapid diagnosis and treatment in hematological malignancies (Kyle et al., 2003; Mai et al., 2007). And PCCP indicated that for those with the selectively abnormal results of laboratory testing, the clinical condition would be comprehended by detailed medical chart review, history taking or physical examination of the patient by the clinical pathologist; the aberrant data were interpreted in combination with clinical presentation of the subject, and patient-oriented laboratory comment was consequently added and validated in the laboratory reports with the liaison service. The laboratory consultation had been mentioned over the decades (Burke et al., 1995), and the concept and clinical practice of such active laboratory consultation had been promoted and performed in 1997 by the clinical pathologist in Japan (Kuwajima et al., 1997; Kuwajima et al., 1998). It was reported that in a 5-year interval, a sum of 303 patient-oriented laboratory comments were reported on the selective hematological and chemical testing results, and $33 \%$ of them had an effect on clinical diagnosis and treatment (Kuwajima et al., 1998). These results were similar to our data which showed that approximately $40 \%$ of the laboratory reports with PCCP had an influence on the clinical diagnosis and therapeutic strategy.

One recent investigation provided detailed evidence about the help-seeking to diagnostic interval in hematological malignancies, and it was shown that the unacceptable prolongation of help-seeking to diagnostic interval was observed in certain hematological cancers such as myelomas and some lymphomas (Howell et al., 2013; Lyratzopoulos et al., 2013). In such malignancies, the initial symptoms could be vague and hence overlooked, leading to the repeated consultations of general practitioner and prolonged interval before referral to the specialties (Lyratzopoulos et al., 2012). In our results, clinical presentations of the individual were rechecked with appropriate interpretation for the aberrant results of hematological laboratory with PCCP and the help-seeking to diagnostic interval was obviously shortened especially in CLL, MM and MDS/MPN, in comparison with the study described above. PCCP should play an essential role in the avoidance of diagnostic delay for hematological cancers, leading to less incident complications and better outcomes.

Our study had some major limitations. First, the study design was retrospective and the case number was extremely limited with some common varieties of hematological cancers and inadequate study interval, making it difficult to determine whether the effect of PCCP on the shortened help-seeking to diagnostic interval in hematological cancers was statistically significant. Additionally, whether the overall survival was improved with the assistance of PCCP remained ascertained. It could be expected that, however, PCCP should shorten the help-seeking to diagnostic interval and promoted the timely diagnosis of hematological cancers, thus avoiding the poor prognosis (Kariyawasan et al., 2007).

In summary, it was seemingly that PCCP prevented diagnostic delay in hematological malignancies via shortening the help-seeking to diagnostic interval, particularly in CLL, MM and MDS/MPN. PCCP was considered to play an essential role in the promptly diagnostic establishment in hematological malignancies for those who were newly diagnosed.

\section{References}

Abdel-Rahman M, Stockton D, Rachet B, et al (2009). What if cancer survival in Britain were the same as in Europe: how many deaths are avoidable? Br J Cancer, 101, 115-24.

Elliss-Brookes L, McPhail S, Ives A, et al (2012). Routes to diagnosis for cancer-determining the patient journey using multiple routine data sets. Br J Cancer, 107, 1220-26.

Friese CR, Abel GA, Magazu LS, et al (2009). Diagnostic delay and complications for older adults with multiple myeloma. Leuk Lymphoma, 50, 392-400.

Howell DA, Smith AG, Roman E (2008). Help-seeking behaviour in patients with lymphoma. Eur J Cancer Care, 17, 394-403.

Howell DA, Smith AG, Jack A, et al (2013). Time-to-diagnosis and symptoms of myeloma, lymphomas and leukaemias: a report from the Haematological Malignancy Research 


\section{Chih-Chun Chang et al}

Network. BMC Hematol, 13, 9.

Irfan SM, Bhurgri Y (2009). Clinico-pathological features and outcomes in chronic phase chronic myeloid leukemia patients treated with hydroxyurea. Asian Pac J Cancer Prev, 10, 591-94.

Kariyawasan CC, Hughes DA, Jayatillake MM, et al (2007). Multiple myeloma: causes and consequences of delay in diagnosis. QJM, 100, 635-40.

Kuwajima M (1997). Clinical laboratory consultation in the general hospital. Rinsho Byori, 45, 727-31.

Kuwajima M (1998). Active laboratory consultation for the clinical supporting system in the general hospital. Rinsho Byori, 46, 994-8.

Kyle RA, Gertz MA, Witzig TE, et al (2003). Review of 1027 patients with newly diagnosed multiple myeloma. Mayo Clin Proc, 78, 21-33.

Lyratzopoulos G, Neal RD, Barbiere JM, et al (2012). Variation in number of general practitioner consultations before hospital referral for cancer: findings from the 2010 national cancer patient experience survey in England. Lancet Oncol, 13, 353-65.

Lyratzopoulos G,Abel GA, McPhail S, et al (2013). Measures of promptness of cancer diagnosis in primary care: secondary analysis of national audit data on patients with 18 common and rarer cancers. Br J Cancer, 108, 686-90.

Mai Y, Qi P, Xu Y, et al (2007). Clinical and laboratory features of newly diagnosed multiple myeloma: a retrospective, single-centre analysis. Chin Med J, 120, 1727-9.

Burke MD (1995). Clinical laboratory consultation. Clin Chem, 41, 1237-40.

Ong F, Hermans J, Noordijk EM, et al (1995). Presenting signs and symptoms in multiple myeloma: high percentages of stage III among patients without apparent myelomaassociated symptoms. Ann Hematol, 70, 149-52

Summerfield GP, Carey PJ, Galloway MJ, et al (2000). An audit of delays in diagnosis and treatment of lymphoma in district hospitals in the northern region of the United Kingdom. Clin Lab Haematol, 22, 157-60.

Thomson CS, Forman D (2009). Cancer survival in England and the influence of early diagnosis: what can we learn from recent EUROCARE results? Br J Cancer, 101, 102-9. 